\title{
THE ANNUAL MEETING IN CHICAGO
}

The sixty-sixth annual meeting of the American Mathematical Society was held at the Conrad Hilton Hotel in Chicago, Illinois, on January 27,28 , and 29, 1960. Several other mathematical organizations also held meetings during the period January 27-30. These were the Mathematical Association of America, the Society for Industrial and Applied Mathematics, and the National Council of Teachers of Mathematics. About 1300 persons registered, including 875 members of the American Mathematical Society.

The thirty-third Josiah Willard Gibbs Lecture was presented by Professor Julian Schwinger of Harvard University, with President McShane presiding. It was a memorable occasion and set a brilliantly high standard for the series. The title of his lecture was Quantum field theory, and was delivered at 8:00 P.M. on Wednesday, January 27 in the Grand Ballroom.

The presidential address was given by Professor Richard Brauer of Harvard University on Thursday, January 28, at 1:00 P.M. in the Boulevard Room. Professor Brauer's lecture was entitled On finite groups and their characters, and Professor L. R. Wilcox of the Illinois Institute of Technology and Chairman of the Committee on Arrangements presided. At the conclusion of the presidential address there was a business meeting, followed by the presentation of the Cole Memorial Prize. On this occasion there were two recipients: Professor Serge Lang of Columbia University and Professor Maxwell Rosenlicht of the University of California, Berkeley. Professor Lang's award was based on his paper in the Annals entitled Unramified class field theory over function fields in several variables. Professor Rosenlicht's award was made on the basis of two articles in the same journal entitled Generalized Jacobian varieties, and $A$ universal mapping property of generalized Jacobians. The Selection Committee consisted of Professor John Tate, Chairman, and Professors Harish-Chandra and Reinhold Baer. Professor A. A. Albert of the University of Chicago presided.

By invitation of the Committee to Select Hour Speakers for Annual and Summer Meetings, there were two addresses. Professor A. S. Besicovitch of Cambridge University and the University of Pennsylvania spoke on Tangential properties of sets of arcs of infinite linear measure at 2:00 P.M. on Wednesday, January 27. Professor Paul Rosenbloom of the University of Minnesota addressed the Society on Partial differential equations with a constant coefficient at 2:00 P.M. 
on Friday, January 29. Both lectures were in the Boulevard Room, and Professor Tibor Rado of The Ohio State University presided at the first, while Professor C. L. Dolph presided at the second.

Sessions for contributed papers, twenty-seven in all, were held in six groups of four and one group of three at various times throughout the meeting. The number of contributed papers was about $35 \%$ larger than the previous maximum achieved in any meeting of the Society. In fact, so keen was the membership on making presentations that sessions actually had to be held in the evening and the meeting did not end until late in the evening of Friday, January 29an unprecedented event.

The current plan adopted by the Society of listing the times at which various papers are to be presented puts an additional burden on the presiding officers. Consequently, the Society is all the more grateful to the following individuals who assisted in this task. Professors Everett Pitcher, H. B. Curry, C. E. Sealander, J. W. Green, E. Snapper, Harvey Cohn, R. F. Rinehart, Zeev Nehari, F. A. Ficken, Marshall Hall, Orville Harrold, M. R. Hestenes, Gale Young, Roger Lyndon, Joseph LaSalle, Victor Shapiro, J. G. Hocking, W. T. Reid, H. P. Thielman, R. H. Oehmke, R. F. Williams, Herman Rubin, L. W. Johnson, L. C. Young, George M. Ewing, Wallace Givens, and I. N. Herstein.

Abstracts of the 222 papers presented in person and the additional 62 papers presented by title appear in the Notices of the American Mathematical Society for December, Issue No. 43, and February, Issue No. 44.

The trustees met on Thursday, January 28.

A Business Meeting of the Society was held in the Boulevard Room at 2:00 P.M., Thursday. President McShane presided. The Secretary expressed the thanks of the Society to the local arrangements committee, and otherwise reported briefly on the affairs of the Society. In response to several queries from the floor concerning the desirability of moving the Annual Meeting back to the period between Christmas and New Year, and from hotels back to universities, the Secretary reported that these matters had already been discussed by the Council and that the President had been asked to appoint a committee to look in to these matters in light of the experience of the past several years.

Following the Business Meeting, the Cole Prize in Algebra was awarded to Professors Serge Lang and Maxwell Rosenlicht. Professor Lang's prize-winning memoir was Unramified class field theory over function fields in several variables, which appeared in the Annals 
of Mathematics, vol. 54, no. 2 (1956) pp. 285-325; those of Professor Rosenlicht were Generalized Jacobian varieties, Annals of Mathematics, vol. 59, no. 3 (1954) pp. 505-530, and $A$ universal mapping property of generalized Jacobians, Annals of Mathematics, vol. 66, no. 2 (1957) pp. 80-88. Professor A. A. Albert made the presentation.

The Council met on Thursday afternoon, January 28, 1960.

The Secretary announced the election of the following one hundred persons to ordinary membership in the Society:

Mr. J. P. Andersen, University of Nebraska;

Professor E. L. Aparo, University of Rome;

Mr. J. R. Ballard, National Bureau of Standards, Boulder, Colorado;

Mr. J. D. Barbour, Raytheon Company, Bristol, Tennessee;

Mr. J. R. Baugh, University of California, Los Angeles;

Mr. R. L. Bohuslov, Oakland City College;

Mr. E. E. Bosman, Pocono Mountain Joint Schools, Cresco, Pennsylvania;

Mr. F. W. Brundage, International Business Machines Corporation, Owego, New York;

Sister Mary Z. Brunell, O.P., Albertus Magnus College;

Professor R. N. Buchal, Mathematics Research Center, U. S. Army, University of Wisconsin;

Professor C. R. Burton, San Diego State College;

Mr. M. L. Cantor, Brooklyn Junior High School 263, Brooklyn, New York;

Mrs. Elizabeth Z. Chapman, Midwestern University Research Association, Madison, Wisconsin;

Mr. N. D. Cohen, Burroughs Corporation, Pasadena, California;

Mr. R. L. Cooley, Purdue University;

Mr. W. A. Crabtree, Jr., Austin Peay State College, Clarksville, Tennessee;

Mr. John de Pillis, University of California, Berkeley;

Professor Jovan Djurić, Northeastern University;

Mr. R. B. Doggett, Air Force Cambridge Research Center, Hanscom Field, Bedford, Massachusetts;

Professor A. T. Dou, Universidad de Madrid;

Dr. T. A. Dwyer, Marycliffe, Glencoe, Missouri;

Mr. James Dyer, Computer Control Company, Los Angeles, California;

Mr. R. J. Engert, Autonetics, Los Angeles, California;

Mr. Samuel Feder, Bulova Research and Development Laboratories, Woodside, New York;

Mr. R. H. Follett, U. S. Army Signal Engineering Agency, Arlington, Virginia;

Professor Martin Fox, Michigan State University;

Professor Avner Friedman, University of Minnesota;

Professor C. B. Gass, DePauw University;

Mr. Allan Gewirtz, Waldorf Industrial Controls, New York, New York;

Mr. A. G. Gluckman, Curtiss-Wright Corporation, Newark, New Jersey;

Dr. Nicholas Glyptis, Multi-tron Laboratory, Westchester, Illinois;

Professor L. A. Goodman, University of Chicago;

Professor J. W. Graham, University of Waterloo;

Mr. A. J. Haltmaier, Oceanside Senior High School, Oceanside, New York;

Mr. S. C. Hanna, Sylvania Electric, West Roxbury, Massachusetts; 
Mr. R. B. Harwitz, International Business Machines, Wilmington, Delaware;

Mr. Ernest Heilberg, CORG, Technical Operations, Inc., Fort Monroe, Virginia;

Professor B. W. Helton, University of Utah;

Mr. J. A. Hildebrandt, University of Tennessee;

Mr. J. J. Hole, Kerr-McGee Oil Industries, Inc., Oklahoma City, Oklahoma;

Mr. E. H. Hopper, Alabama Polytechnic Institute;

Professor O. J. Huval, Southwestern Louisiana Institute;

Mr. C. K. Im, Chungnam University, Taejun, Korea;

Mr. B. G. Isen, Pennsylvania Mutual Life Insurance Company, Philadelphia, Pennsylvania;

Mr. W. H. Jamison, Montana State College;

Captain F. D. Johnson, Wentworth Military Academy, Lexington, Missouri;

Professor Edgar Karst, Brigham Young University;

Mr. Gabriel Klambauer, Assumption University of Windsor;

Dr. L. H. Koopmans, Sandia Corporation, Albuquerque, New Mexico;

Mr. A. F. Koupe, Jr., Westinghouse Electric Corporation, Pittsburgh, Pennsylvania;

Professor Daniel Koval, Atlantic Union College;

Miss Lorraine D. Lavallee, University of Massachusetts;

Dr. R. M. Lewis, New York University;

Mr. C. M. Lobron, International Business Machines, Philadelphia, Pennsylvania;

Mrs. Irmgard Luneburg, American Optical Company, Southbridge, Massachusetts;

Mr. R. C. McCarty, Boeing Airplane Company, Seattle, Washington;

Mr. K. M. McCrimmon, Reed College;

Mr. M. A. MacLeod, Baird-Atomic, Inc., Cambridge, Massachusetts;

Mrs. Arlene B. Macedo, Johns Hopkins University;

Mr. P. A. Martino, University of Kansas;

Dr. J. C. Mathews, Iowa State University;

Mr. J. R. Meachem, Palm Beach Junior College, Lake Worth, Florida;

Miss Valerie Miké, Bell Telephone Laboratories, New York, New York;

Mr. J. P. Miller, General Motors Corporation, Milwaukee, Wisconsin;

Mr. M. K. Nestell, Southern Missionary College;

Mr. J. W. O'Brien, North Providence High School, North Providence, Rhode Island;

Professor J. R. Padró, University of Puerto Rico;

Professor R. L. Page, Nasson College;

Dr. E. A. Pflumm, Republic Aviation Corporation, Farmingdale, New York;

Professor Rose M. Ring, Boston College;

Professor Wendy S. Robertson, University of Kansas;

Mr. R. E. Roth, St. Bonaventure University;

Mr. R. J. Runge, California Research Corporation, La Habra, California;

Professor L. W. Rutland, Jr., University of Colorado;

Mr. W. A. Salmi, Rockford College;

Mr. D. A. Sanchez, University of Michigan;

Dr. Moshe Shimrat, University of Michigan;

Mr. J. D. H. Sibley, Georgetown University;

Professor A. L. Siebenlist, Southern Oregon College;

Mr. J. A. Simpson, University of Arizona;

Mr. R. C. Sine, University of Illinois;

Mr. K. E. Smith, Lenkurt Electric Company, San Carlos, California;

Mr. T. M. Spencer, Sperry Utah Engineer Laboratory, Salt Lake City, Utah;

Professor M. M. Stanišić, Purdue University; 
Professor S. R. Sukhn, National School of Mathematical Sciences, Beirut, Lebanon; Professor L. F. Takács, Columbia University;

Mr. E. R. Tomer, IBM Watson Laboratory, New York, New York;

Mr. H. H. Torriani, University of Oklahoma;

Dr. Y.-O. Tu, International Business Machines Research Center, Yorktown Heights, New York;

Mr. D. H. Underwood, Whitman College;

Mr. H. N. Ward, Harvard University;

Mr. G. M. Webster, Jersey Production Research Company, Tulsa, Oklahoma;

Professor J. B. Wells, Jr., University of Kentucky;

Mr. K. W. Weston, Waukesha, Wisconsin;

Professor J. C. Wilson, Central College;

Lieutenant J. D. Woods, U. S. Coast Guard, San Juan, Puerto Rico;

Reverend J. S. Wright, Yonkers, New York;

Professor W. S. Wunch, Arizona State University:

Mr. F. O. Wyse, Oregon State College;

Professor Elias Zakon, Assumption University of Windsor;

Dr. N. R. Zitron, Brown University.

It was reported that two hundred and thirty-one persons had been elected to membership on nomination of institutional members as indicated:

Alabama Polytechnic Institute: Mr. D. E. Dupree.

University of Alberta: Mr. D. M. Aitken, Mr. S. G. Mohanty, Mr. James Riddell, Mr. J. R. Trollope.

University of Arizona: Mr. R. T. Fritsche.

University of British Columbia: Mr. W. R. Gordon, Mr. J. E. Vinson, Mr. Roy Westwick.

Brooklyn College: Mr. Wolfe Snow, Mr. Robert Greenblatt.

Brown University: Mr. R. L. Fosdick, Mr. M. A. Hayes, Mr. Hung-ta Ho, Mr. D. W. Jonah, Professor G. F. Newell, Mr. Skarphedinn Palmason, Mr. H. M. Roberts, Mr. T. H. Teng, Mr. T.-C. Woo, Mr. Bohyun Yim, Mr. W. P. Ziemer.

University of California, Berkeley: Mr. Andrew Astromoff, Mr. M. L. Bandy, Mr. R. F. Barnes, Jr., Mr. R. H. Cornell, Mr. J. E. Fenstad, Mr. F. J. Forelli, Jr., Mr. H. J. Keisler, Mr. E. G. K. Lopez-Escobar, Mr. Puey-chong Lum, Mr. D. D. Mann, Mr. W. D. Maurer, Mr. Tamio Ono, Mr. H. J. Pohlmann, Mr. H. L. Resnikoff, Mr. J. H. Simons, Mr. Alvin Swimmer, Mr. A. T. Vasquez.

University of Californis, Davis: Mr. L. L. Berryman.

University of California, Los Angeles: Mr. A. O. Allen, Mr. J. I. Derr, Mr. H. A. Gindler, Mr. D. H. Gottlieb, Mr. T. G. McLaughlin, Mr. Burton Rodin, Mr. E. F. Steil, Mr. R. J. Weiss, Mr. G. S. McCarty, Jr.

California Institute of Technology: Mr. R. D. Bercov, Mr. R. L. Held, Mr. D. W. Willett.

Case Institute of Technology: Mr. M. E. Conway.

Catholic University of America: Sister Kathleen Ann Bellcourt, Sister Margaret Grace Elsey, Captain W. H. Lake.

University of Chicago: Professor C. A. Abuauad, Mr. D. L. Barnett, Mr. Alphonse Buccino, Mr. J. A. Eagon, Miss Elaine D. Goldman, Mr. D. G. Heller, Mr. J. B. Minkus, Professor Helene A. Rasiowa, Mr. Fred Richman, Mr. R. I. Sandler, Mr. F. W. Schneider, Mr. D. M. Shafer, Mr. R. M. Solovay, Mr. P. F. G. Stanek, Mr. W. A. Strauss. 
University of Colorado: Mr. Herbert Walum.

Cornell University: Professor Alfred Aeppli, Miss Dorothea M. Bamberger, Mr. C. A. Berger, Mr. P. L. Gale, Miss Patricia A. Cundall, Mr. W. R. Gavin, Mr. D. G. Kuehner, Mr. N. F. Laubscher, Mr. B. J. Moran, Mr. D.-F. Neu, Mr. G. L. Sward, Mr. Y.-F. Wong.

De Paul University: Mr. A. Z. Czarnecki.

Harpur College: Mr. J. A. Higgins, Jr.

Johns Hopkins University: Mr. R. N. Draper, Mrs. Anne C. W. Greene, Mr. M. H. Hyman, Miss Ann W. Penn, Lieutenant A. M. Potter, Jr., Mr. A. I. Thaler, Mr. H. J. Tramer.

University of Illinois: Mr. A. W. Addison, Miss Renu Chakravarti, Mr. J. W. Corbett, Mr. R. A. DeMarr, Sister Madeleine Sophie (Drufenbrock), Mr. C. W. Gear, Mr. H. S. Hahn, Mr. D. S. Geiger, Mr. L. S. Levy, Mr. D. A. Moran, Professor Subramonier Parameswaran, Mr. H. C. Tung, Mr. Sunardi Wirjosudirdjo.

Lehigh University: Mr. G. F. Heck, II, Mr. R. W. Packard, Mr. R. E. Roberts, Jr.

University of Maryland: Mr. J. S. Kim, Mr. S. R. Kraft, Mr. R. J. McConahy, Mr. F. T. Metcalf, Mr. E. C. Young.

Massachusetts Institute of Technology: Dr. D. J. Benney, Mr. Martin Billik, Mr. D. S. Kahn, Mr. L. N. Patterson.

University of Miami: Mr. R. L. Kelley, Mr. E. V. Nunnally, Mr. C. D. Sikkema.

Michigan State University of Agriculture and Applied Science: Mr. G. T. Chartrand, Mr. R. L. Gantos, Mr. G. V. Rowell, Mr. M. S. Rowin, Mr. Ernst Schwandt, Mr. J. L. VanIwaarden.

University of Michigan: Mr. R. H. Abraham, Professor R. J. Newman, Mr. E. R. Suryanarayan, Mr. E. J. Vought.

University of Missouri: Mr. George Burke, Mr. E. B. Lassen.

Montana State University: Mr. William Kirkpatrick, Mr. I. K. Yale.

University of Nebraska: Mrs. Mildred L. Gross.

University of New Hampshire: Mr. E. S. Folias.

New York University: Mr. G. S. de Souza A'vila, Professor A. T. Dou, Mr. Reuben Hersh, Mrs. Sondra O. Jaffe, Mr. Charles Kahane, Mr. G. H. Kuby, Miss Jeannette Salkind.

University of North Carolina: Mr. A. L. Deal, III, Mrs. Sarah G. Glover, Mr. P. H. Jessner, Mr. R. S. Johnson, Miss Ruth E. Kurtzweil, Mr. K. G. Witz.

Ohio State University: Mr. W. E. Coppage, Mr. R. D. Dixon, Mr. J. E. Duemmel, Mr. Marcellus Duffy, Jr., Mr. D. J. Schaefer, Mr. A. L. Smith.

Ohio Wesleyan University: Mr. M. D. Magree.

University of Oklahoma: Mr. A. S. Davis, Mr. B. G. Hodges, Mr. L. L. Koontz, Jr.

University of Oregon: Mr. D. W. Bailey, Mr. C. H. Chicks, Mr. J. Q. Denton, Mr. Burke Zane.

Pennsylvania State University: Mr. J. P. Alford, Mr. K. D. Magill, Jr., Mr. T. E. Mott.

University of Pennsylvania: Mrs. Ruth S. Beck, Mr. D. J. Parker, Mr. Robert Rich, Miss Nina Schertz, Mr. A. M. Smuckler, Professor R. J. Wagner, Mr. Paul Weinberg.

Purdue University: Mr. D. C. Boes, Mr. D. B. Coleman, Mr. S. E. J. Johnsen, Mr. Siyaram Srivastava, Mr. K. V. Venkataraman, Mr. R. R. Welland.

Rice Institute: Mr. J. R. Cannon, Mr. B. F. Jones.

Rutgers, The State University: Mr. J. D. Cowie, Mr. R. B. DiFranco, Mr. R. M. Fesq, Jr., Mr. L. B. Sklar.

Sacramento State College: Mr. T. D. Cavanagh. 
College of St. Thomas: Mr. R. J. La Voi.

Stanford University: Mr. R. K. Bhattacharyya, Mr. D. F. Brauch, Mr. M. J. Donohoe, Mr. B. L. Ehle, Mr. K. T. Hahn, Mr. L. D. Meeker, Mr. Leon Nower, Mr. F. B. Thiess, Mr. D. G. Wendin.

University of Tennessee: Mr. C. H. Edwards, Jr., Mr. G. F. Hampton, Mr. M. C. McCord.

University of Texas: Mr. J. T. Darwin, Jr., Mr. E. I. Deaton, Mr. J. A. Dyer, Mr. H. G. Ellis, Mr. T. L. Hayden, Mr. C. A. Greathouse.

Tulane University: Mr. C. J. Hightower, Mr. W. C. Holland, Mr. Kazimierz Urbanik. University of Utah: Mr. H. C. Wiser.

Vanderbilt University: Mr. D. H. Engelhardt.

University of Washington: Mr. J. M. Albarez de Araya, Mr. J. W. Armstrong, Mr. D. C. Correa, Mr. A. J. Froderberg, Mr. James Michelow, Mr. S. S. Mitra, Mr. Mario Petrich, Mr. L. A. Wold.

Washington State University: Mr. A. L. Peressini.

Wayne State University: Mrs. Linda F. Foulis, Professor J. G. Sowul.

University of Wisconsin: Professor R. N. Braae, Mr. R. F. Brown, Mr. Charles Ehrenpreis, Mr. R. A. Gaggioli, Mr. E. C. Ingraham, Mr. D. E. Julie, Miss Nora E. Lazier, Mr. B.-F. Lee, Mr. D. G. Moursund, Miss Joan E. Rand, Mr. B. W. Romberg, Mr. C. P. Seguin, Mr. H. B. Skerry, Dr. Wojciech Slowikowski, Mr. D. J. Sterling, Miss Mudite Upesleja, Mr. Jim Van Buskirk, Mr. Robert Vermes, Mr. D. C. West, Mr. L. E. Whitford.

Yale University: Mr. Christopher Bingham, Sister Mary Zachary Brunell, Mr. H. F. Kreimer, Jr., Mr. Fumiyuki Maeda, Mr. J. E. Simpson, Mr. D. A. Smith.

Yeshiva University: Mr. Jonah Mann, Mr. C. R. Patt, Mr. H. Z. Senter.

The Secretary announced that the following had been admitted to the Society in accordance with reciprocity agreements with various mathematical organizations: Dansk Matematisk Forening: Professor H. E. Skovgaard; Deutsche Mathematiker Vereinigung: Dr. Günther Goes, Dr. Edmund Hlawka, Dr. J. A. Nitsche, Professor E. H. Thoma; London Mathematical Society: Dr. W. N. Everitt, Mr. Bernard Fishel, Dr. P. J. Higgins, Dr. A. P. Robertson, Dr. D. C. Russell, Dr. James Wiegold; Polskie Towarzystwo Mathmatyczne: Dr. A. A. Kosinski, Dr. S. G. Mrowka; Schweizerische Mathematische Gesellschaft: Professor Dr. L. Bieberbach; Mathematical Society of Japan: Mr. Masahisa Adachi, Mr. Masahiko Atsuji, Mr. Sin Hitotumatu, Mr. Eishi Hongo, Mr. Seiiti Husino, Mr. C.-J. Hsu, Mr. Yoshio Inagaki, Mr. Kazuo Ishiguro, Mr. Ryosuke Iwahashi, Mr. Takao Kakita, Professor Yoshie Katsurada, Mr. Tatsuo Kawata, Mr. Masanori Kishi, Mr. Yusaku Komatu, Professor Hajime Kosugi, Mr. Hisao Mizumoto, Professor Yosio Muto, Mr. Mitsuru Nakai, Mr. Masao Narita, Professor Masami Ogawara, Mr. Laoru Ohkubo, Professor Tanjiro Okubo, Mr. Mitsuru Ozawa, Mr. Shiroshi Saito, Professor Takashi Shibata, Mr. Nobuo Shimada, Mr. Kenichi Shiraiwa, Mr. Wasao Sibagaki, Mr. Noboru Suzuki, Mr. Mitsuo Sugiura, Mr. Tadashi Tanaka, Mr. Hisahiro Tamano, Mr. Fumio 
Tanimoto, Mr. Yoshihiro Tashiro, Mr. Ishihara Tetsuo, Mr. TetsuoNagata, Mr. Akira Tominaga, Mr. Tosiro Tsuzuku, Mr. Tadashi Ugaheri, Professor Minoru Urabe, Mr. Hiroshi Watanabe, Mr. Kiyosi Yamaguti, Dr. Taro Yoshizawa.

It was reported that Professor Walter H. Durfee had represented the Society at the inauguration of William Spencer Litterick as President of Keuka College; Professor William R. Transue had represented the Society at the inauguration of David Alexander Lockmiller as President of Ohio Wesleyan University; Professor J. Crawford Polley had represented the Society at the inauguration of Ralph Alexander Morgan as President of Rose Polytechnic Institute; Professor Morris Kline had represented the Society at the One Hundredth Anniversary of the Cooper Union; Dean Mina Rees and Professor Garrett Birkhoff had represented the Society at a Conference on Research Goals at Worcester Polytechnic Institute.

The following committee appointments of the President were reported: as a Committee to Consider By-Laws Concerning Election and Term of Executive Director: S. Bochner, J. H. Curtiss, A. H. Taub, J. W. T. Youngs, Chairman; as tellers for the 1959 election: Robert J. Blattner, L. J. Paige; as a Committee to Select Candidates for the Executive Committee of the Council for 1960: A. A. Albert, R. P. Dilworth, G. B. Price; as an Arrangements Committee for the Summer Meeting at Michigan State University in 1960: H. L. Alder, W. E. Deskins, J. S. Frame, F. Herzog, L. M. Kelly, M. L. Tomber, Gordon L. Walker, C. P. Wells, J. W. T. Youngs; to the Committee to Select Speakers for Far Western Meetings: J. L. Kelley; as Chairman of the Organizing Committee for Summer Institutes: R. S. Phillips.

The Secretary reported that the following have accepted invitations to deliver hour addresses before the Society: P. A. Smith, Summer Meeting, 1959; J. H. C. Whitehead, November, 1959, Wayne University; H. J. Reyser, November, 1959, Wayne University; John Todd, November, 1959, University of Southern California; Trevor Evans, November, 1959, Winston-Salem, North Carolina; A. S. Besicovitch, P. R. Rosenbloom, Annual Meeting, 1960;T. S. Motzkin, February, 1960, University of Arizona; F. B. Fuller, April, 1960, Berkeley, California; S. Karlin, June, 1950, Missoula, Montana.

The Secretary reported that Reciprocity Agreements have been made with the Mathematical Societies of Japan and Australia.

JoHN W. GREen, Secretary

J. W. T. Youngs, Associate Secretary 